$\mathrm{Oz}$

$1-1-2014$

\title{
Topological Phenomenology of Space: Architecture as Roots of Infinity
}

Peter Magyar

Kansas State University

Follow this and additional works at: https://newprairiepress.org/oz

Part of the Architecture Commons

(c) (1) $\Theta$

This work is licensed under a Creative Commons Attribution-Noncommercial-No Derivative Works 4.0 License.

\section{Recommended Citation}

Magyar, Peter (2014) "Topological Phenomenology of Space: Architecture as Roots of Infinity," Oz: Vol. 36. https://doi.org/10.4148/2378-5853.1532

This Article is brought to you for free and open access by New Prairie Press. It has been accepted for inclusion in Oz by an authorized administrator of New Prairie Press. For more information, please contact cads@k-state.edu. 


\section{Peter Magyar}

Ideas mature, but interestingly enough, they never get old! It is also notable how are they born. In my case, two elements brought them forward: one question and an admirably unique surrounding. Let me begin with the latter one. In the beginning of my so-called mid-career, I had the great opportunity to live, practice, and teach in Africa! For almost four years we lived in Zaria, an ancient Nigerian town, with a modern university outside its medieval walls. It is impossible to list all the enlightening influences one encounters in a strange, yet deep-rooted culture! Worthy of at least another article, the Muslim socio-religious ideas, the abundance of the reddish lateritemud, and the inherent human ability to build, produced an incredibly homogeneous physical and ideological environment. Addressing here only the physical one, it was an almost dream-like uniformity between the ground, the walls, and roofs of the self-built villages! So no wonder, when in the process of designing a modern, but traditional, house in the city of Ife, another old town, to the question, what is the condition of spatial continuity, the subconsciously preprogrammed answer was: surface continuity! This obvious recognition of an element, visual and dominantlypresent everywhere, fermented further meditations on that subject and the idea of the "Spaceprint" was born.

Returning to the subject of this article, "Phenomenology" comes from the Greek "phainomenon" = that which appears, and "logos" = study. "(It) is the philosophical study of the structures of experience and consciousness."

We will stop here, and I will explain the willful marriage tha I forced "topology" and "phenomenology" into. Jean Piaget, the late Swiss philosopher and clinical psychiatrist, after hundreds of actual tests with children of very young age, in his book, The Child's Perception of Space, made the statement that our psyche, our consciousness, is organized by topological principles. Also, Maurice Merleu-Ponty, one of the fathers of phenomenology, wrote this in his book, The Primacy of Perception: “...if we are seeking to form an idea of, or to understand the essence of, a spatial figure...we must first perceive it. Then we will imagine all the aspects contained in the figure as changed. That which cannot be varied without the object itself disappearing is the essence." ${ }^{2}$ This is a very important, maybe unintentionally topological statement. So, good bye phenomenology, hello topology!

Paul Valery, in "Eupalinos ou l'Architecte" wrote, "Music and Architecture force us, to transcend in thought that which they would seem, in reality, to imply; they rest in the middle of this world like monuments of another world; or like scattered instances of structure and time which are not products of human beings, but of basic forms and laws." ${ }^{3}$

Doing a competition for "A House for Johann Sebastian Bach," I made the following assumption: music can be perceived as a "knot" on the infinite line of time, while architecture is a "spatial-loop (-system)" on the infinite surface of the Earth, which separates space and non-space.

So, the "knotters" and the "loopers" are doing very similar service to mankind: to bring forth what is un-hearable and in-visible for most everybody, except for those whose existence is rewarded and burdened with this self-imposed duty.
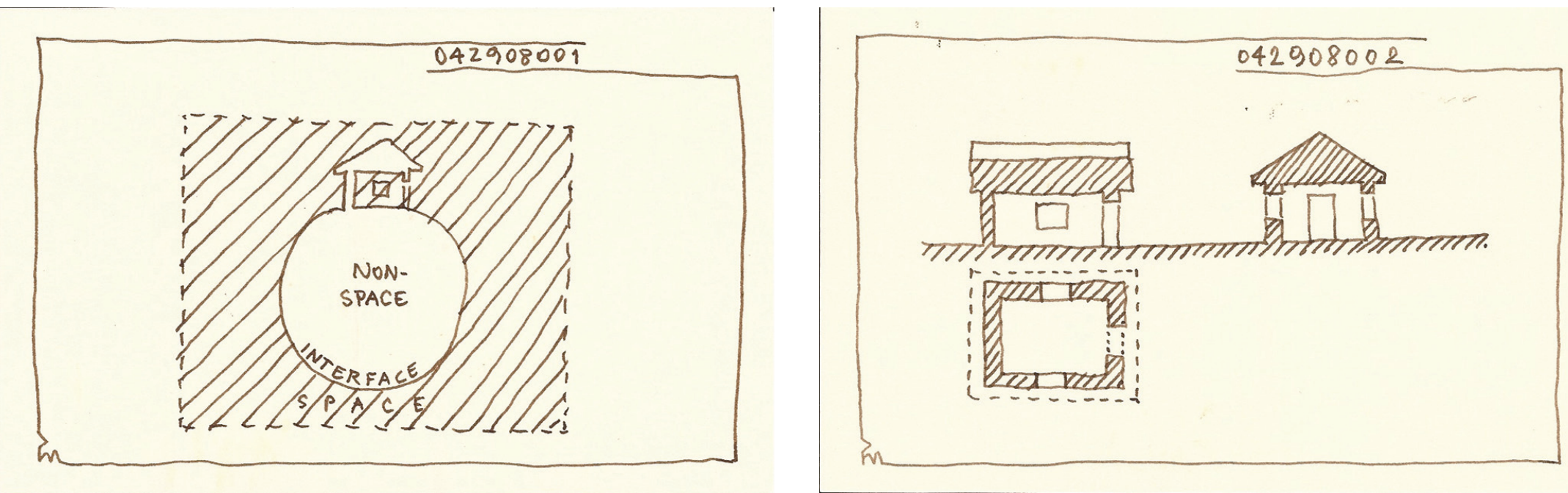

Figure 2. Spaceprint 
Again, knots and loops are very much in the language of topology, so let us see what they mean for our investigation.

Topology is the youngest- but fastest-developing, and one of the most important aspects of mathematics and geometry. In my studies, at this time, I apply only one simplified branch of topology, the visual topology. This kind of topological transformation does not conserve the proportions, measurements, or directions, but only the continuity and neighborhood relations of the surface are to be un-changed. "Rubber sheet-geometry," because it deals with that property that an object retains under deformation, specifically bending, stretching, and squeezing, but not breaking or tearing. Since topology by its nature is not scale-specific, topological transformations can be applied from micro to macro scale (e.g., industrial design, urban design).

We can then continue with the following assertions: if we free the boundary conditions from the measurable (metric) restrictions while applying topological transformations to them, we produce the "Topologically Equivalent Model" and we can call it "General Spaceprint" (the "essence" of Merleau-Ponty). Every "Particular Spaceprint" is reducible to one "General Spaceprint" but from one "General Spaceprint" unlimited number of "Particular Spaceprints" can be generated. Based on Jean Piaget's research, ${ }^{4}$ I forward the hypothesis: there might exist a correlation between a so called Biologically Coded Formal Preference and the similarity of the General and Particular Spaceprints, which similarity I call "Topological Isomorphy."5

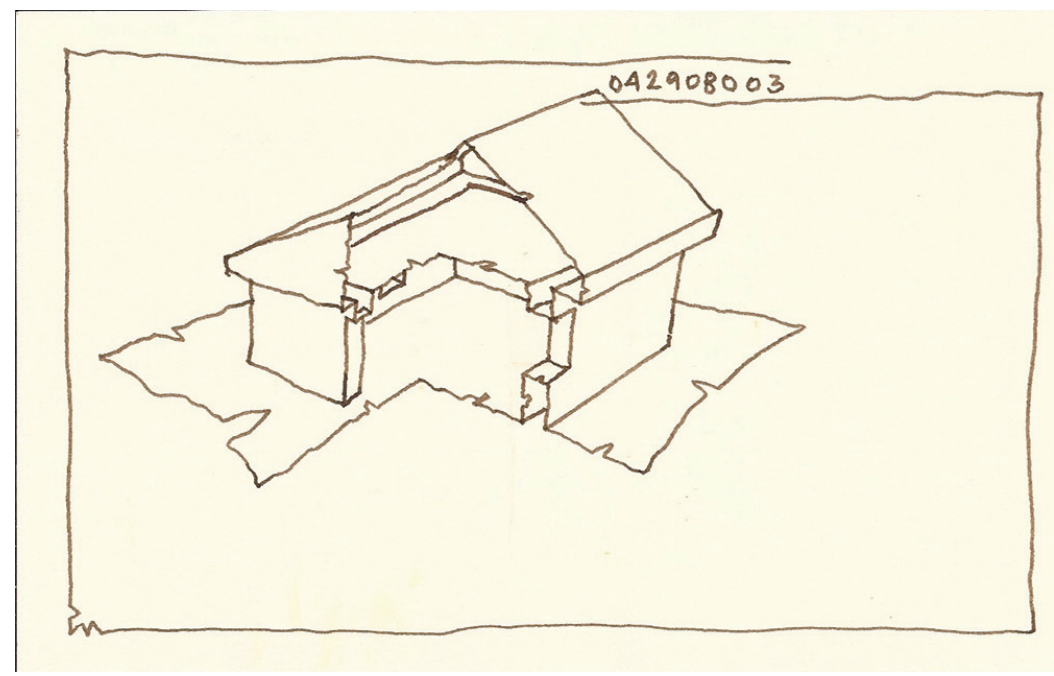

Figure 3. Particular Spaceprint

According to general practice in architecture, intentionally or unintentionally, the shapes of the "Spaceprints" are conceived and articulated by design and realized through construction. Therfore architecture as a design process is the planning of, and as a product is the execution of, appropriate changes in the surfaces or "Spaceprints".

Let us nowlook at the drawings. On our diagrams, the upper surface represents the stretched, flattened surface of the Earth, including the similarly-handled external surfaces of buildings. The "tubes" stand for the openings (windows, doors), connecting the internal surfaces of the rooms (in this case the "bubbles") with the "infinite," or, at least, immeasurable Earth-face, and the different rooms with each other. Observations and applications of this method for many years, led to the following simplified, drawn-manifesto:

\section{Figure 1}

- Architecture deals with two essential domains: space and non-space. - Space contains non-space.

- The interface of these domains is named "Spaceprint"; it simultaneously describes the localized shapes of both space and non-space.

- The condition of spatial continuity is surface continuity.

\section{Figure 2}

- Common behavior in the practice of architecture, that we are talking about space, yet non-space is drawn.

\section{Figures 3 and 4}

- "Particular Spaceprints" could describe the shapes of an object and as well as of space.

- Intentionally or unintentionally, these spaceprints are imagined in architectural design, and materialized in building construction.

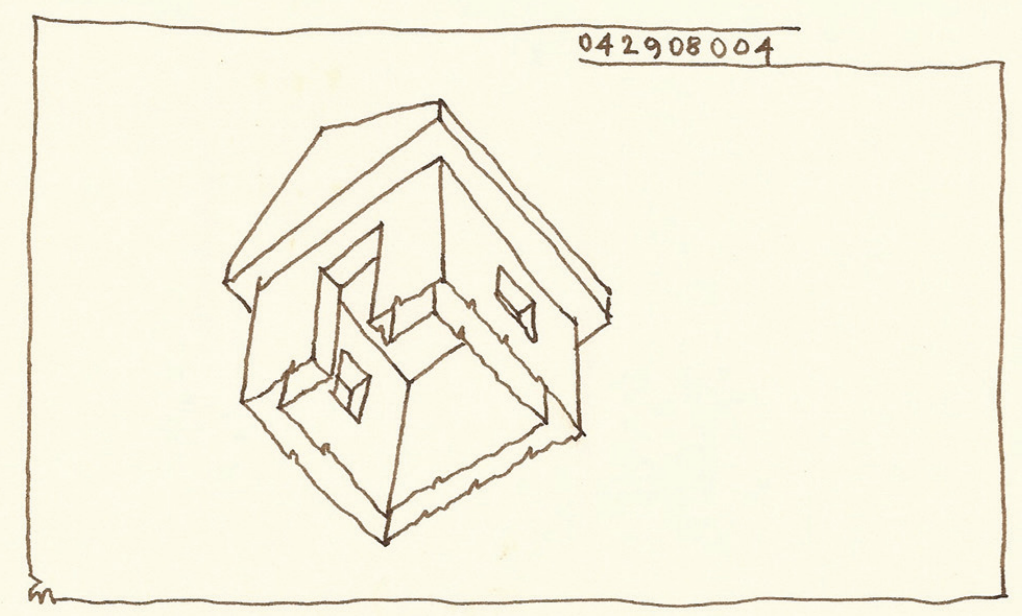

Figure 4. Particular Spaceprint 


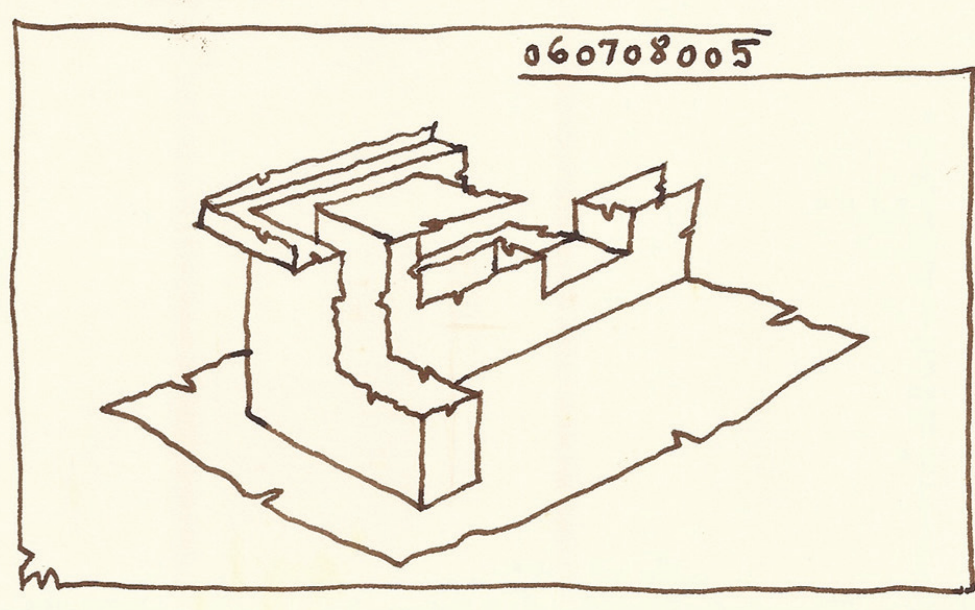

Figure 5. Spaceprint Fragment

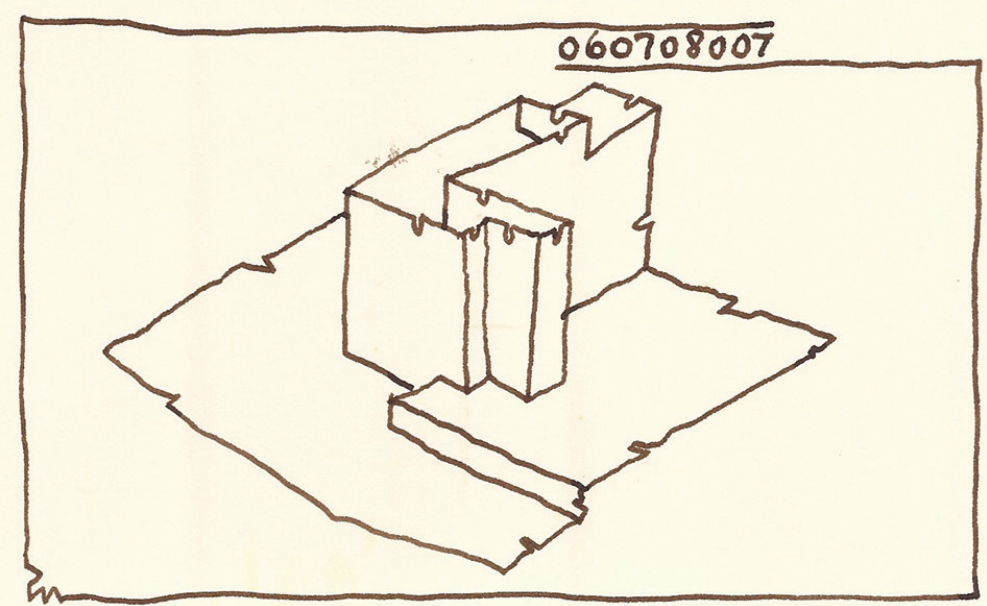

Figure 7. Spaceprint Fragment

- In construction, one distinguishes surface providers and surface holders.

- Traditional materials are bifunctional, while new structures show distinct separation of these two functions.

\section{Figures 5 through 8}

- "Spaceprint Fragments" describe surface strategies, changes in the surface usually denote changes in

\section{Figures 9 and 10}

- Applying to the "Particular Spaceprints" the rules of topology, where metric properties (measurements, directions, proportions) are not preserved, only the neighborhood relationships, we can create the "General Spaceprints."

Every "Particular Spaceprint" can be transformed to only one "General Spaceprint", but the latter could be reversed to numberless versions of the former.

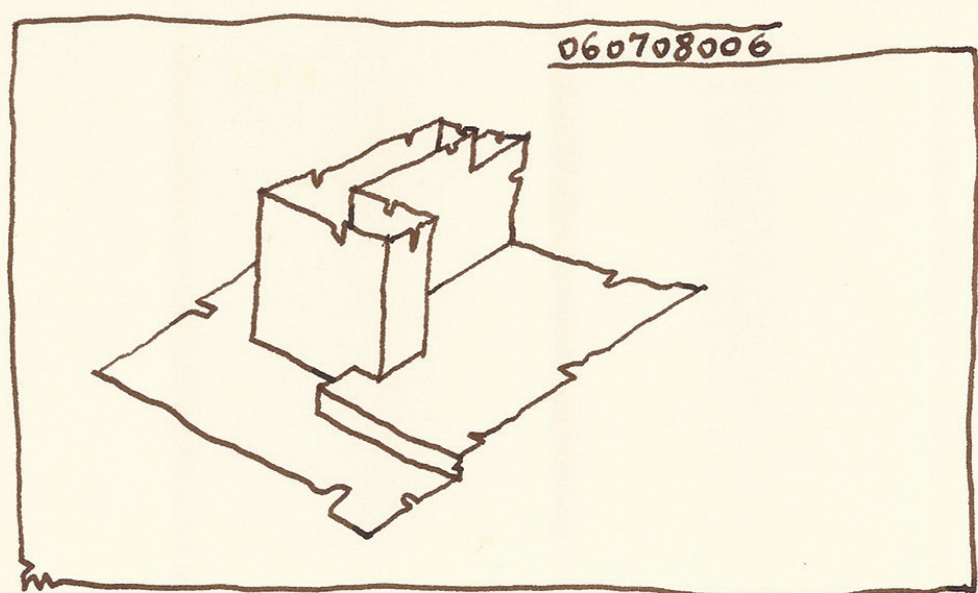

Figure 6. Spaceprint Fragment

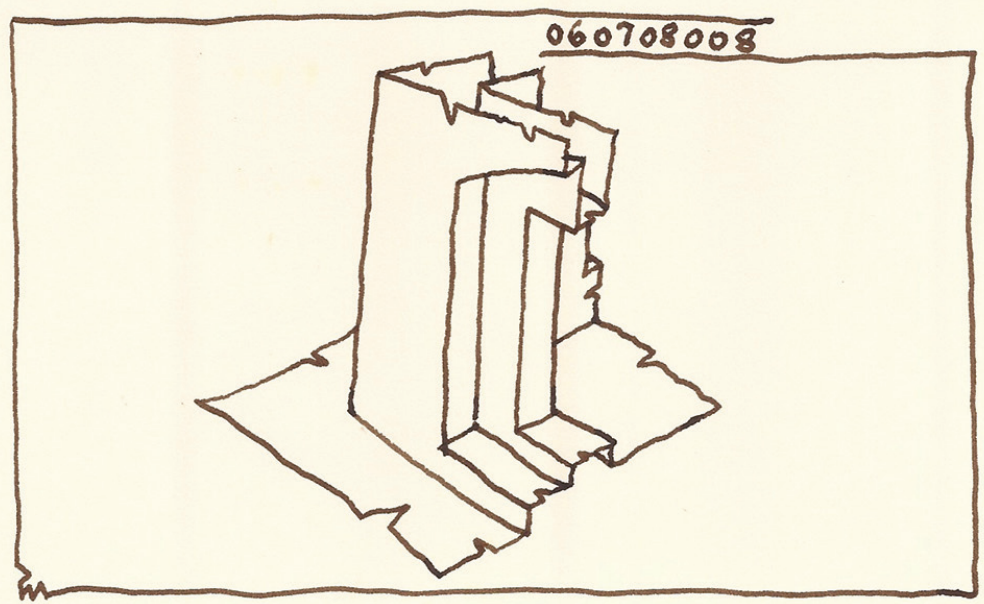

Figure 8. Spaceprint Fragment

- Topological transformations are often called "rubber-sheet-geometry, hence the rounded shapes and tube-like openings. These representing the doors, windows, and other openings, seamlessly connect internal surfaces with the external ones.

\section{Figures 11 and 12}

- Applying the infinite pliability of these imaginary surfaces, the "General Spaceprints" can be trans- formed and the result is the "Simplified General Spaceprint” or SGSP.

(If I may indulge in the patience of my readers, let me recommend them to visit the following You Tube video: http://www.youtube.com/ watch?v=JhxyTozjHE)

This reduction method resulted in a relatively simple tool, which denotes spatial structure, and as such, could be applied in analytical and compara- 


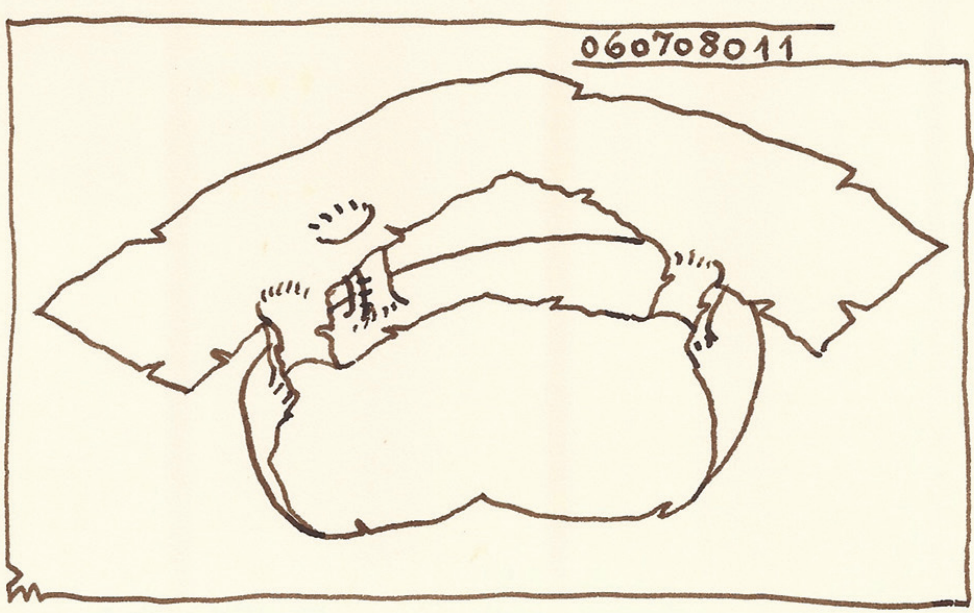

Figure 9. General Spaceprint

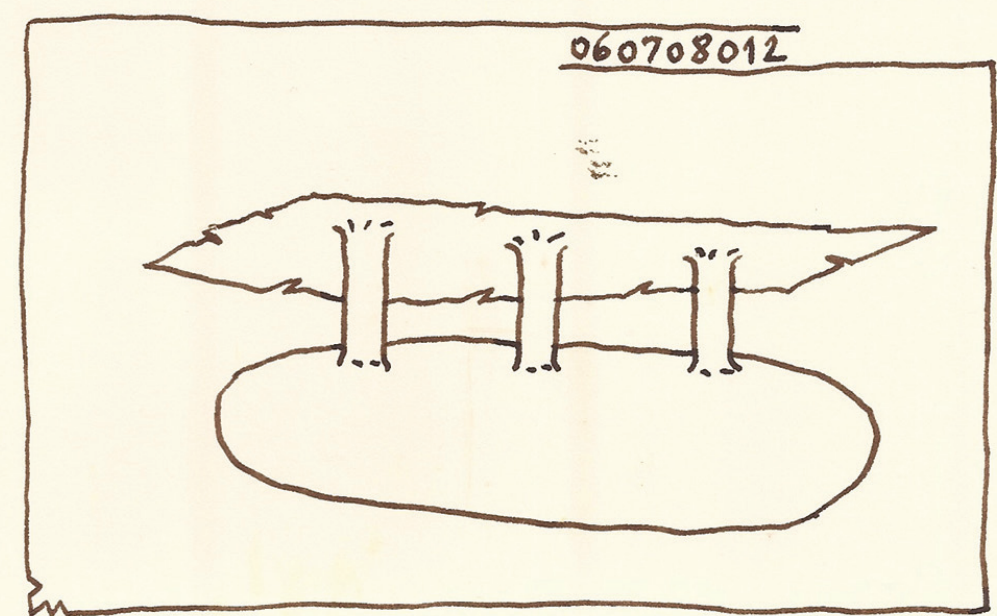

Figure 11. Simplified General Spaceprint

tive studies of space in architecture. As a shorthand depiction, they could be used as a programming tool, (in a SGSP format) or in the "bubbles and tubes" version, they can reveal spatial relationships of any building. These characteristics enable this process, to be applied for the establishment of spatial typologies.

The following drawings are but the first batch of a collection of Simplified General Spaceprints and General

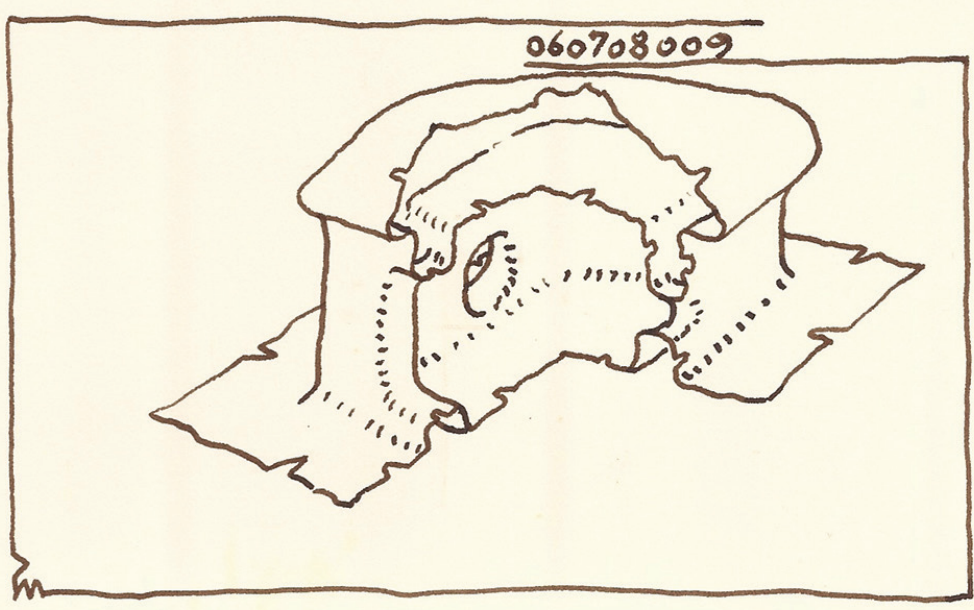

Figure 10. General Spaceprint

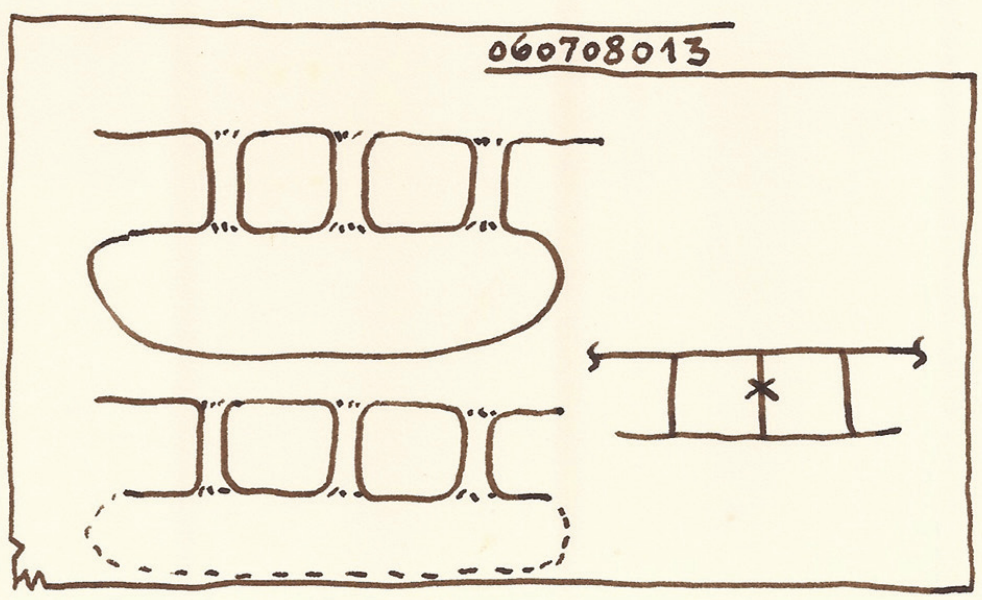

Figure 12. Simplified General Spaceprint

ing of individual spaces, or even the external appearance of a building is concerned. However, under these seemingly simple space-perceptions, the incredibly complex spatial structure is hidden. So far, the Spaceprint method might be the only one that is able to reveal the complex system of spatial loops, which are present even in a seemingly simple building. The following drawings are excerpts from the works of some of the students in my "Spaceprint Seminar," when they had to map out their respective residencies, in both of the Simplified General Spaceprint, or in the General Spaceprint forms. As a final test of their analytical abilities, based on my SGSP drawing, they had to visualize and draw the General Spaceprint of Antonio Palladio's well-known Villa La Rotonda. This centrally symmetrical building was chosen due to its seemingly simple and repetitious spatial system, only to be surprised by the inherent complexity of the spa- 
tial structure. If in the earlier shown examples one didn't recognize the obvious visual symbolism between these General Spaceprints, now it is inevitable to not to see them as root-systems, the Roots of Infinity $=\sqrt{ } \infty .($ Figures 13-17)

This interpretation of Architecture reveals omnipresent, but newly formulated aspects for the enrichment and ritualization of our spatial perception. With the application of the theoretical tools of reduction and topological transformation, the infinite expansion of space, at least cognitively, can be comprehended. Myriad attempts were made to discover a believable and shared symbolism of Architecture since the lost secrets of the Renaissance. The obvious but lost symbol is its cosmic scale! The perception of the presence of gravity and spatial infinity also very rarely happens. One can easily imagine, in the case of even one, or many, high-rise buildings, how complex, and literally deep-rooted this connection and symbolic representation of anchoring infinity could become. We can talk about low-, medium-, high-, super-, and hyperintensity degrees; and the number of mangrove-like "roots" in the cities (= "root-fields") Once understood that even the simplest building has an intimate, and mostly hidden relationship to infinity, architects suddenly became the new priests and shamans of our society, because they do what their awed predecessors were doing: speaking to and with the Cosmos!
1. See: http://en.wikipedia.org/wiki/Phenomenology_(philosophy)

2. Merleau-Ponty, Maurice. The Primacy of Perception (Chicago: Northwestern University Press, 1964) p.70.

3. Valery, Paul. "Eupalinos ou l'Architecte" in Euvres, vol.2 (Paris: Gallimard, 1960).

4. Piaget, J. at al., The Child's perception of Space (Chicago: Northwestern University Press, 1956) pp. 21 and 55 .

5. Magyar, Peter. Thought Palaces (Amsterdam Architectura \& Natura Press, 1999).

\section{Image Credits}

All images by the author, except where noted

\section{Acknowledgements}

The author would like to express his appreciation to Wesley Gross and Elias Logan, the editors of $\mathrm{Oz} 36$, for their constructive comments and assistance. I am indebted to the students, credited on their figures, for their permission to use the drawings, and for those whose work didn't get into the journal, but worked diligently and with great curiosity towards the topic. Finally, Professor Ray Streeter's photo of the Villa La Rotonda lent a beautiful aura to this issue of $O z !$

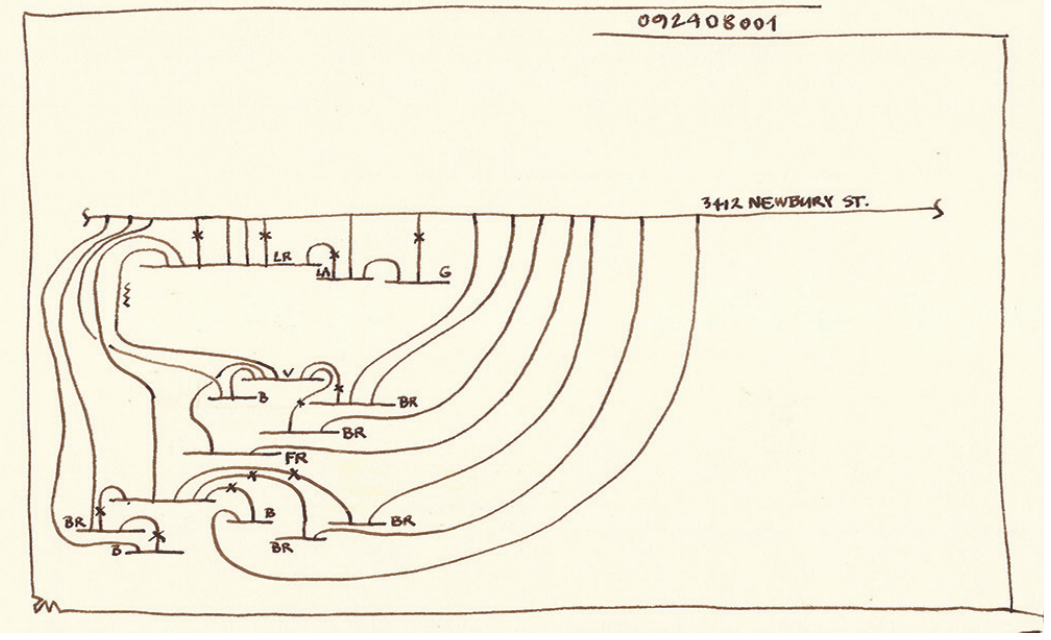

Figure 13. Simplified General Spaceprint of a residence

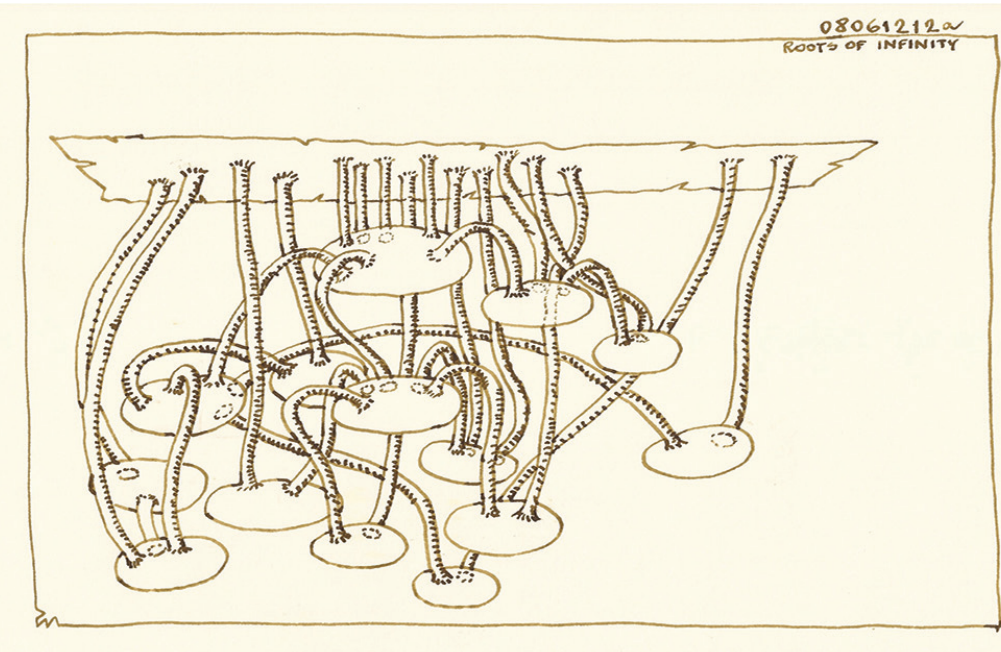

Figure 14. General Spaceprint of residence above

\section{Notes}




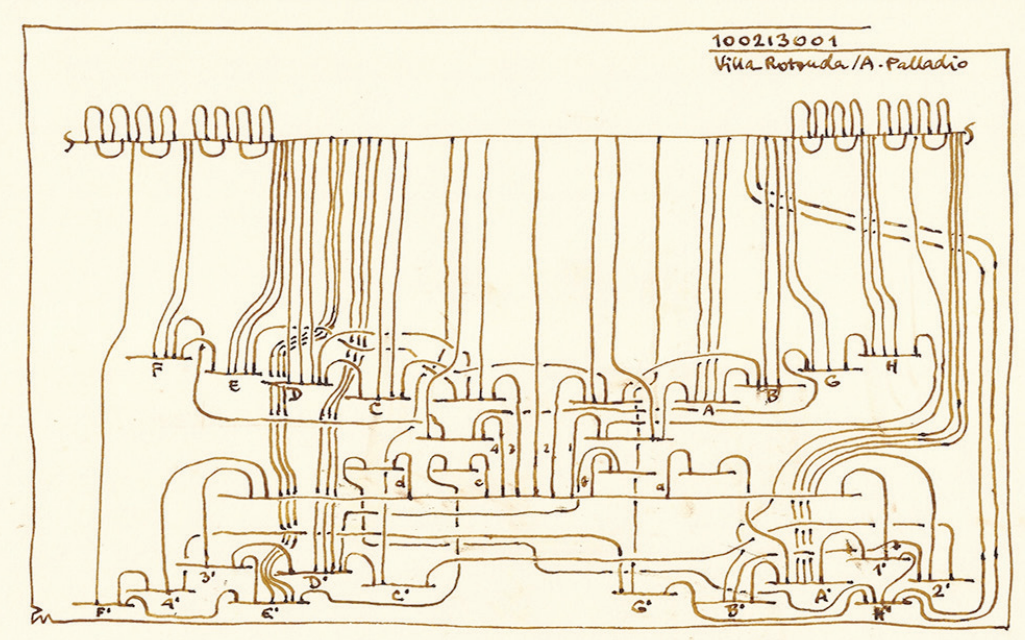

Figure 15. Simplified General Spaceprint of the Villa Rotunda

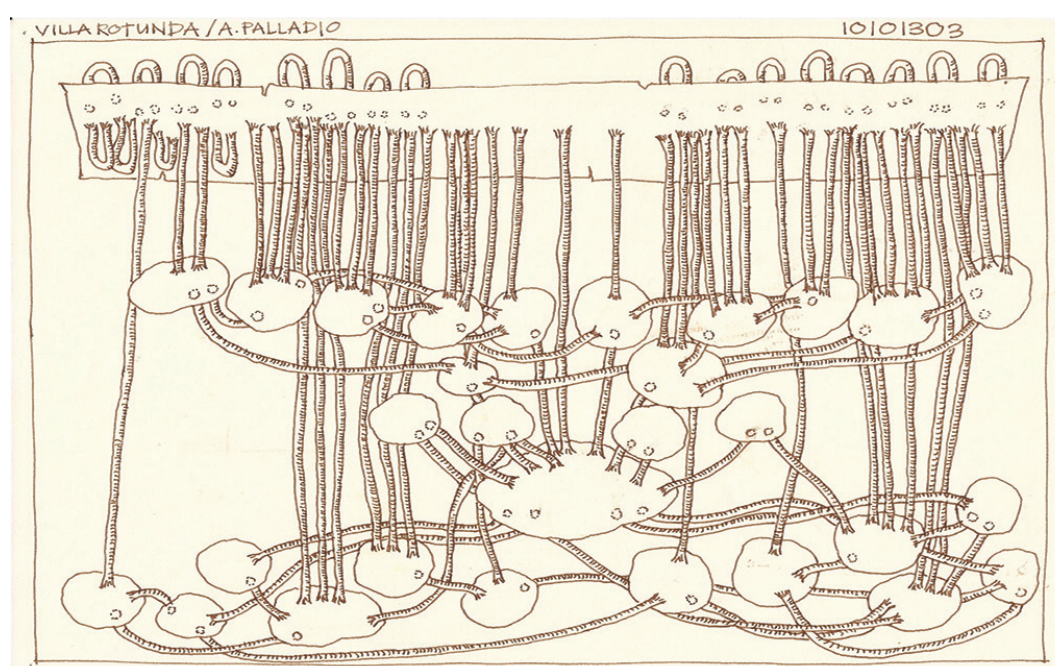

Figure 16. General Spaceprint of the Villa Rotunda, by Kirby Thomas

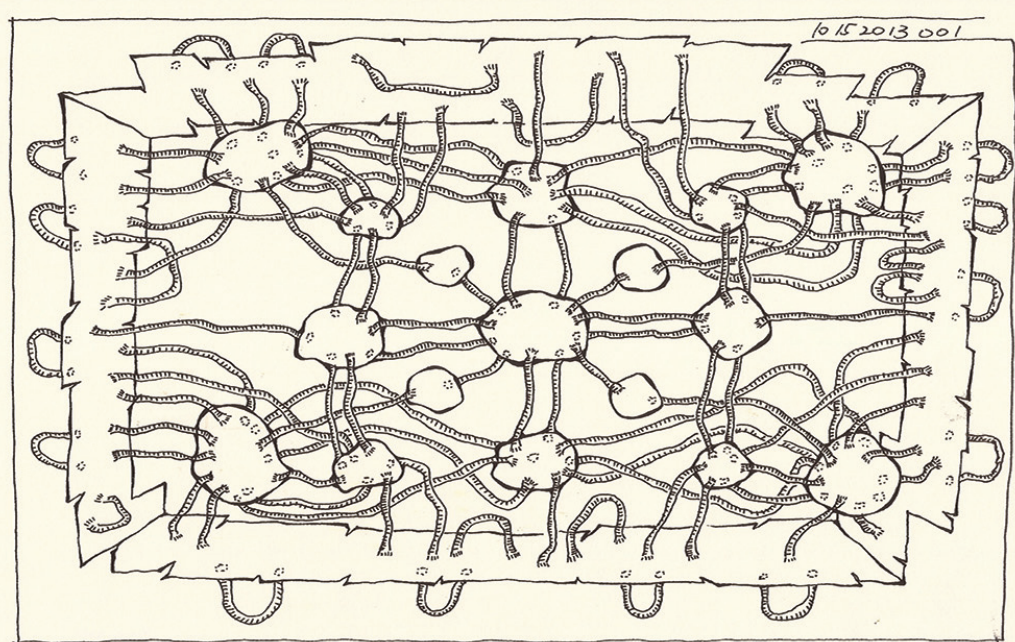

Figure 17. Variation on the Villa Rotunda, by Ying Zhu

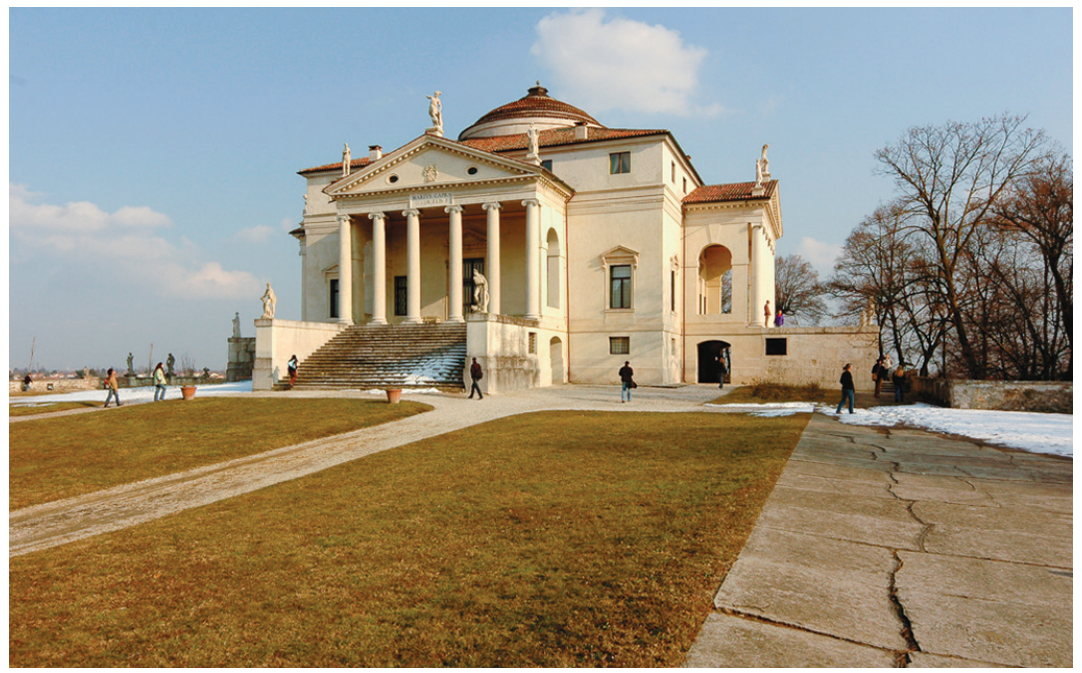

Figure 18. Villa Rotunda, photo by Ray Streeter 